\title{
Tat-indoleamine 2,3-dioxygenase 1 elicits neuroprotective effects on ischemic injury
}

\author{
Jung Hwan Park ${ }^{1, \#}$, Dae Won Kim ${ }^{2, \#}$, Min Jea Shin ${ }^{1, \#}$, Jinseu Park ${ }^{1}$, Kyu Hyung Han ${ }^{1}$, Keun Wook Lee ${ }^{1}$, Jong Kook Park ${ }^{1}$, \\ Yeon Joo Choi ${ }^{1}$, Hyeon Ji Yeo ${ }^{1}$, Eun Ji Yeo ${ }^{1}$, Eun Jeong Sohn ${ }^{1}$, Hyoung-Chun Kim ${ }^{3}$, Eun-Joo Shin ${ }^{3}$, Sung-Woo Cho , \\ Duk-Soo Kim ${ }^{5}$, Yong-Jun Cho ${ }^{6}$, Won Sik Eum ${ }^{1, *}$ E Soo Young Choi, \\ ${ }^{1}$ Department of Biomedical Science and Research Institute of Bioscience and Biotechnology, Hallym University, Chuncheon 24252, \\ ${ }^{2}$ Department of Biochemistry and Molecular Biology, Research Institute of Oral Sciences, College of Dentistry, Gangneung-Wonju National \\ University, Gangneung 25457, ${ }^{3}$ Neuropsychopharmacology and Toxicology Program, BK21 PLUS Project, College of Pharmacy, Kangwon \\ National University, Chunchon 24341, ${ }^{4}$ Department of Biochemistry and Molecular Biology, University of Ulsan College of Medicine, Seoul \\ 05505, ${ }^{5}$ Department of Anatomy and BK21 Plus Center, College of Medicine, Soonchunhyang University, Cheonan 31538, ${ }^{6}$ Department of \\ Neurosurgery, Hallym University Medical Center, Chuncheon 24253, Korea
}

It is well known that oxidative stress participates in neuronal cell death caused production of reactive oxygen species (ROS). The increased ROS is a major contributor to the development of ischemic injury. Indoleamine 2,3-dioxygenase 1 (IDO-1) is involved in the kynurenine pathway in tryptophan metabolism and plays a role as an anti-oxidant. However, whether IDO-1 would inhibit hippocampal cell death is poorly known. Therefore, we explored the effects of cell permeable Tat-IDO-1 protein against oxidative stress-induced HT-22 cells and in a cerebral ischemia/reperfusion injury model. Transduced Tat-IDO-1 reduced cell death, ROS production, and DNA fragmentation and inhibited mitogen-activated protein kinases (MAPKs) activation in $\mathrm{H}_{2} \mathrm{O}_{2}$ exposed $\mathrm{HT}-22$ cells. In the cerebral ischemia/ reperfusion injury model, Tat-IDO-1 transduced into the brain and passing by means of the blood-brain barrier (BBB) significantly prevented hippocampal neuronal cell death. These results suggest that Tat-IDO-1 may present an alternative strategy to improve from the ischemic injury. [BMB Reports 2020; 53(11): 582-587]

\section{INTRODUCTION}

Indoleamine 2,3-dioxygenase 1 (IDO-1) is a heme-containing

${ }^{*}$ Corresponding authors. Soo Young Choi, Tel: +82-33-248-2112; Fax: +82-33-248-3202; E-mail: sychoi@hallym.ac.kr; Won Sik Eum, Tel: +82-33-248-3221; Fax: +82-33-248-3202; E-mail: wseum@hallym.ac.kr ${ }^{\text {\#}}$ These authors contributed equally to this work.

https://doi.org/10.5483/BMBRep.2020.53.11.114

Received 28 May 2020, Revised 16 June 2020,

Accepted 30 June 2020

Keywords: Ischemia, MAPKs, Oxidative stress, Protein therapy, TatIDO-1 enzyme involved in the first step of the kynurenine pathway in tryptophan metabolism and is expressed in response to interferon gamma (IFN- $\gamma$ ) stimulation in the cortex, hippocampus, and various cells, including neurons, astrocytes, macrophages, and microvascular endothelial cells (1-3). The kynurenine pathway finally generates kynurenic acid and quinolinic acid. Quinolinic acid causes excitotoxicity and neuronal cell death, whereas kynurenic acid has antioxidant properties $(4,5)$. IDO-1, a unique cytosolic enzyme, exerts powerful antioxidant effects by means of free radical scavengers $(4,6,7)$. Over expressed human IDO-1 by gene transfection significantly protects endothelial cell against damage from oxidative stress and lung transplant ischemia/reperfusion injury in an animal model (8). It also protects against atherosclerosis by regulation of $T$ cells in plasmacytoid dendritic cells (9). Although reactive oxygen species (ROS) are important for keeping balance in cellular redox signaling, overproduction of ROS is involved in neuronal diseases including ischemia (10-13). Since ROS play crucial roles in the pathogenesis of this disease, antioxidant protein seems to be a potential therapeutic approach for ischemic injury $(14,15)$.

Mitogen-activated protein kinases (MAPKs) signaling pathways, such as extracellular-signal regulated kinase (ERK), c-Jun $\mathrm{NH} 2$ terminal kinase (JNK), and p38 are associated with cell differentiation, cell proliferation, cell survival, and cell death (16). Even though several studies have reported that oxidative stress-mediated MAPKs activation plays an important role in death-receptor-initiated exogenous and mitochondrial apoptotic pathways as well as neuronal cell death or neurodegenerative disorders (17-19), little is known about the effects of IDO-1 on oxidative stress-mediated neuroprotective effects in hippocampal cells and a cerebral ischemia/reperfusion injury model.

It is recognized that small molecules can transduce into the cell, but larger macromolecules like protein cannot permeate owing to their physicochemical characteristics $(20,21)$. Thus, we fused IDO-1 protein with protein transduction domains (PTD),

ISSN: 1976-670X (electronic edition)

Copyright (C) 2020 by the The Korean Society for Biochemistry and Molecular Biology

(c) This is an open-access article distributed under the terms of the Creative Commons Attribution Non-Commercial License (http://creativecommons.org/licenses/by-nc/4.0) which permits unrestricted non-commercial use, distribution, and reproduction in any medium, provided the original work is properly cited. 
such as Tat peptide, which can allow protein to transduce into cells. In previous studies, we showed that PTD fusion proteins transduced into cells and significantly protected them against various oxidative stress-induced diseases (14, 15, 22, 23). In this study, we investigated whether Tat-IDO-1 inhibits hippocampal cell death in HT-22 cells and a cerebral ischemia/ reperfusion injury model.

\section{RESULTS}

\section{Construction, production, and transduction of recombinant} Tat-IDO-1 fusion protein

As shown in Fig. 1A, we constructed recombinant Tat-IDO-1 and control IDO-1 plasmid. Tat peptide is linked to a human IDO-1 gene to permit transduction of a fusion protein into cells, whereas a control IDO-1 gene was not linked to the Tat peptide. Then, SDS-PAGE and Western blot analysis confirmed the purified fusion proteins, Tat-IDO-1 and control IDO-1, as shown in Fig. 1B. Purified fusion proteins appeared to have the expected molecular weights of 38 and $36 \mathrm{kDa}$, respectively.

To investigate whether a fusion protein can transduce into HT-22 cells, we treated control IDO-1 and Tat-IDO-1 proteins with cells for various times (10-180 min) and concentrations
A

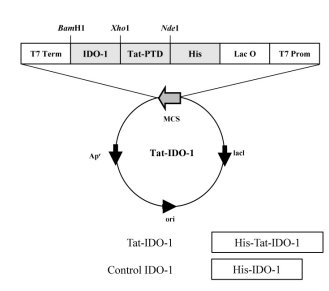

C
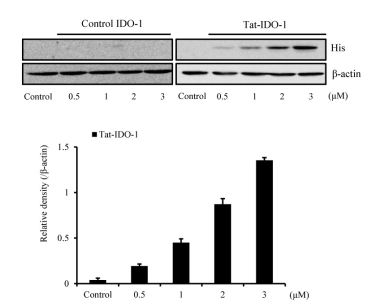
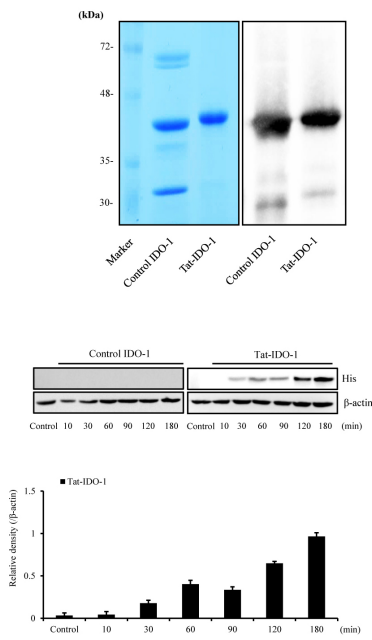

Fig. 1. Construction, purification, and transduction of Tat-IDO-1 protein. Diagrams of Tat-IDO-1 and control IDO-1 protein (A). Purification of Tat-IDO-1 and control IDO-1 protein. We analyzed purified Tat-IDO-1 and control IDO-1 protein using 15\% SDS-PAGE and Western blotting (B). Transduction of Tat-IDO-1 protein into HT-22 cells. The cells were treated with Tat-IDO-1 and control IDO-1 protein $(0.5-3 \mu \mathrm{M})$ for $3 \mathrm{~h}$ or Tat-IDO-1 and control IDO-1 protein $(3 \mu \mathrm{M})$ for various incubation times $(10-180 \mathrm{~min})(\mathrm{C})$. Then, transduced Tat-IDO-1 protein levels were assessed by Western blotting, and the intensity of the bands was measured by a densitometer. Data are repressed as mean $\pm \operatorname{SEM}(n=3)$.
(0.5-3 $\mu \mathrm{M})$. As expected, Tat-IDO-1 protein was detected in a dose- and time-dependent manner (Fig. 1C). Also, transduced protein detected not only stability for up to $36 \mathrm{~h}$ but also distributed both cytosol and nuclei in the cells. In contrast, control IDO-1 was not detected (Supplementary Fig. S1).

\section{Effects of Tat-IDO-1 protein on cell death}

To confirm the effect of Tat-IDO- 1 proteins on HT-22 cell death, $\mathrm{HT}-22$ cells were treated with $1 \mathrm{mM} \mathrm{H}_{2} \mathrm{O}_{2}$ before a cell viability assay (Supplementary Fig. S2). We found that Tat-IDO-1 protein increased cell viability up to $72 \%$ in the presence of the $\mathrm{H}_{2} \mathrm{O}_{2}$. To examine how the Tat-IDO-1 protein affects the cell viability, we investigated cellular toxicity, ROS generation, and DNA damage (Fig. 2A and 2B). We confirmed that TatIDO-1 protein significantly inhibits the cellular toxicities. However, there was no significant difference between $\mathrm{H}_{2} \mathrm{O}_{2}$ and control IDO-1 protein-treated cells.

\section{Effects of Tat-IDO-1 protein on signaling pathways under} oxidative stress

To explore whether there was an association between TatIDO-1 protein and signaling pathways, we investigated apoptosis and MAPK signaling pathways in $\mathrm{H}_{2} \mathrm{O}_{2}$ exposed $\mathrm{HT}-22$ cells. Tat-IDO- 1 protein reduced Bax expression levels more than did the cells treated only with $\mathrm{H}_{2} \mathrm{O}_{2}$. In contrast, $\mathrm{Bcl}-2$ expression levels were increased by Tat-IDO-1 protein. In

A

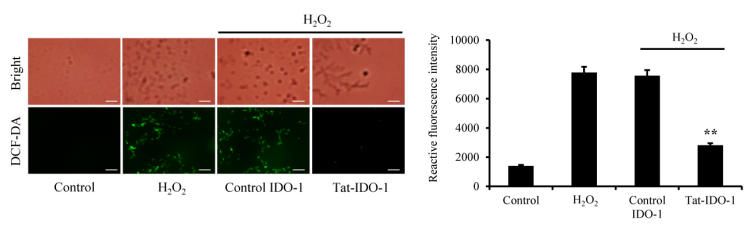

B

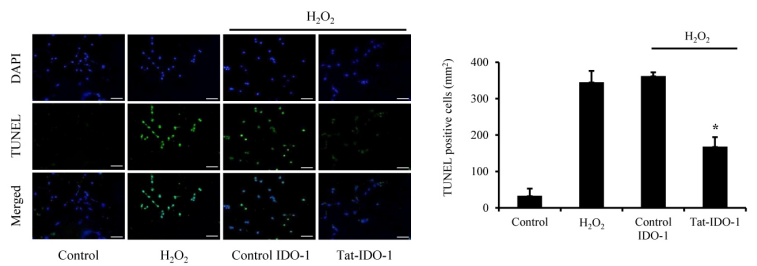

Fig. 2. Effects of Tat-IDO-1 protein against oxidative stress-induced HT-22 cell damage. Tat-IDO-1 $(3 \mu \mathrm{M})$ and control IDO-1 protein (3 $\mu \mathrm{M}$ ) were pretreated with HT-22 cells for $3 \mathrm{~h}$ before treatment with $1 \mathrm{mM}$ of $\mathrm{H}_{2} \mathrm{O}_{2}$. Intracellular ROS levels were measured by DCF-DA staining. Fluorescence intensity was quantified using an ELISA plate reader (A). DNA fragmentation was assessed by TUNEL staining, and quantitative evaluation of TUNEL positive cells were confirmed by cell counting under a phase-contrast microscopy ( $\times 200$ magnification) $(B)$. Scale bar $=50 \mu \mathrm{m}$. ${ }^{* P}<0.05$ and $* * \mathrm{P}<0.01$ compared with $\mathrm{H}_{2} \mathrm{O}_{2}$-treated cells. Data are repressed as mean \pm SEM $(n=3)$. 
addition, Tat-IDO-1 protein reduced cleaved Caspase- 3 and -9 expression levels, whereas control IDO-1 proteins did not affect apoptotic protein expression levels (Fig. 3A and 3B).

As shown in Fig. 3C, phosphorylation of MAPKs (p38, ERK and JNK) expression levels were increased by $\mathrm{H}_{2} \mathrm{O}_{2}$. However, Tat-IDO-1 protein reduced phosphorylation of MAPKs expression levels dose-dependently. Control IDO-1 protein showed patterns similar to those of cells exposed only to $\mathrm{H}_{2} \mathrm{O}_{2}$.

\section{Effects of Tat-IDO-1 protein on a cerebral ischemia/ reperfusion injury model}

We investigated whether Tat-IDO-1 protects against ischemic injury in a cerebral ischemia/reperfusion injury model. Cresyl violet $(\mathrm{CV})$ staining showed that neuronal cell death was markedly increased in the vehicle- or control IDO-1-treated group. However, neuronal cell death was significantly inhibited in the Tat-IDO-1-treated group. Also, ionized calcium-
A

B

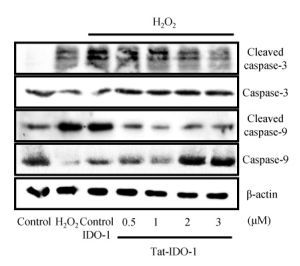

C

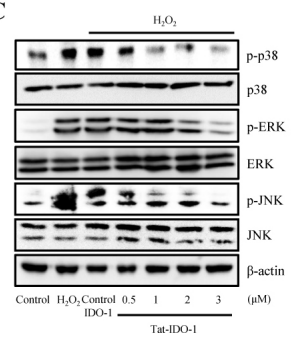

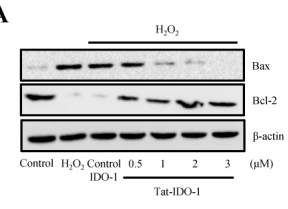
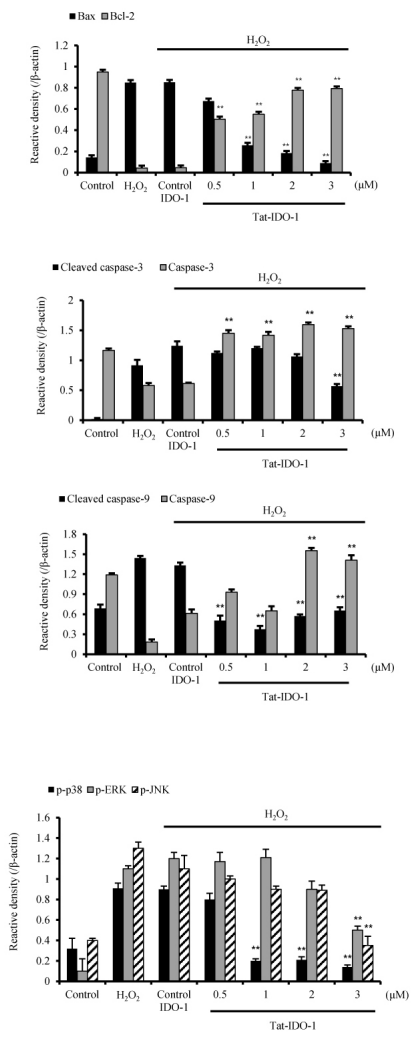

Fig. 3. Effect of Tat-IDO-1 protein on signaling pathways in HT22 cells. Tat-IDO-1 $(0.5-3 \mu \mathrm{M})$ and control IDO-1 protein $(3 \mu \mathrm{M})$ were treated with HT-22 cells for $3 \mathrm{~h}$ before $\mathrm{H}_{2} \mathrm{O}_{2}(1 \mathrm{mM})$. Expression levels Bax and $\mathrm{Bcl}-2$ (A), cleaved Caspase-3 and -9 (B), and MAPKs (C) were detected by Western blotting with indicated specific antibodies. The protein band intensities were measured by densitometer. ${ }^{* * P}<0.01$ compared with $\mathrm{H}_{2} \mathrm{O}_{2^{-}}$ treated cells. Data are repressed as mean \pm SEM $(n=3)$. binding adapter molecule 1 (Iba-1), Fluoro-Jade B (F-JB), and glial fibrillary acidic protein (GFAP) staining were drastically increased in the vehicle- or control IDO-1-treated group. In contrast, Iba-1, GFAP, and F-JB staining were significantly reduced in the Tat-IDO-1-treated group (Fig. 4).

\section{DISCUSSION}

IDO-1 is a key enzyme in tryptophan metabolism and is known to induce the production of metabolite kynurenic acid and quinolinic acid. Kynurenic acid promotes cell survival against oxidative stress, whereas quinolinic acid induces cell death $(24,25)$. Since many studies have demonstrated that IDO-1 protein expression is highly associated with various diseases, including Alzheimer's disease, cancer, and diabetes, IDO-1 is generally known to be a marker of those diseases, and inhibition of IDO protein expression is considered to be a target for various disease therapies $(26,27)$. On the other hand, other studies have shown that IDO-1 expression significantly inhibits oxidative stress-induced cell death by exerting powerful antioxidant functions in cancer, inflammation, and neuronal diseases (28-30). Even though some studies have suggested that the IDO-1 protein can be a therapeutic agent for neuronal and immune-related diseases $(2,3,9)$, the effects of IDO-1 protein in brain ischemia are not fully investigated yet.

PTD has been known as a tool to overcome the delivery limit of a wide array of compounds, such as peptides and proteins in vitro and in vivo $(20,21,31)$ and extensive experiments have shown that PTD fusion protein is transduced into cells and tissues $(14,15,31-35)$. In this study, we showed that cell permeable Tat-IDO- 1 fusion protein is transduced into HT-22 cells. Although Tat-IDO-1 protein transduction ability showed patterns similar to those of other Tat fusion protein
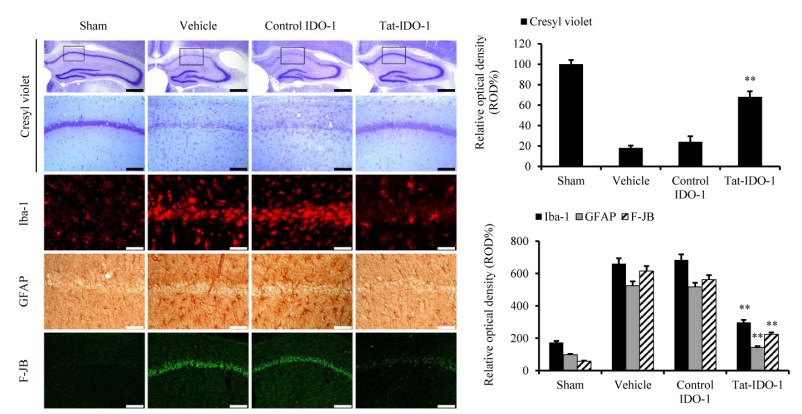

Fig. 4. Effect of Tat-IDO-1 on ischemic injury in a cerebral ischemia/reperfusion model. Neuroprotective effects of transduced TatIDO-1 protein were analyzed by the CV, GFAP, Iba-1, and F-JB immunostaining in the CA1 region of the hippocampus of the gerbil brain 7 days ( $n=10$ per groups) after ischemic injury. Relative numeric analysis of CV, GFAP, Iba-1, and F-JB-positive neurons in CA1 region. Scale bar $=400 \mu \mathrm{m}$ and $50 \mu \mathrm{m}(\mathrm{CV})$, $25 \mu \mathrm{m}$ (GFAP and Iba-1), and $50 \mu \mathrm{m}$ (F-JB). **P $<0.01$, significantly different from the vehicle group. 
studies, the transduction time of Tat-IDO-1 protein is longer than that of other Tat fusion proteins. The difference in transduction time may depend on various factors, such as protein size, polarity, and protein shape.

Excessive production of ROS induced by oxidative stress causes irreversible degeneration of proteins, nucleic acids, and lipids, and ultimately leads to cell death (36). In this study, we showed that transduced Tat-IDO-1 protein markedly inhibited $\mathrm{H}_{2} \mathrm{O}_{2}$-induced cell death, ROS generation, and DNA fragmentation. It has been reported that overexpressed IDO-1 protein inhibited $\mathrm{H}_{2} \mathrm{O}_{2}$-induced cell death, DNA damage, and intracellular ROS levels in an ischemic injury rat model, prevented $\mathrm{H}_{2} \mathrm{O}_{2}$-induced HUVEC cell death, and prevented neuronal cell death by free radical scavengers $(8,37)$. Our finding that Tat-IDO- 1 protein inhibited HT-22 cell death induced by oxidative stress coincides with those reports suggesting that IDO- 1 acts as an antioxidant protein.

Oxidative stress altered the expression levels of Bax and $\mathrm{Bcl}-2$ protein, led to cell death, and expressed high levels of activated cleaved Caspase-9 and Caspase-3 (38, 39). In addition, it is well known that anti-apoptotic protein $(\mathrm{Bcl}-2)$ expression levels were reduced and pro-apoptotic protein (Bax) expression levels were increased under excessive oxidative stress $(38,40)$. Our data also showed that Tat-IDO-1 protein regulated apoptotic protein expression levels, including Bax, Bcl-2, cleaved Caspase-9 and Caspase-3.

It is well known that MAPKs (p38, ERK, and JNK) signaling pathways are highly associated with oxidative stress-induced cell death (41-43). Other studies have shown that overexpression of IDO-1 protein inhibited the activation of Akt and MAPKs signaling pathways and regulated apoptotic protein expression levels in neuronal cells under excessive oxidative stress $(44,45)$. Our results showed the same patterns, indicating that Tat-IDO-1 protein inhibits neuronal cell death by regulation of apoptosis, Akt, and MAPKs signaling pathways under oxidative stress.

Since it has been reported that ROS is a major risk factor in ischemic injury and plays crucial roles in the pathogenesis of ischemia/reperfusion injury $(13,36)$, we examined whether Tat-IDO-1 protein protects against ischemic injury in a cerebral ischemia/reperfusion injury animal model. Several studies have demonstrated that activated astrocytes and microglia cells are highly associated with ischemic injury; these cells were increased in brain ischemia, and their reactivities were increased in the hippocampus and led to neuronal cell death by release of pro-inflammatory cytokines and neuroinflammatory response (46-49). Also, Liu (2007) demonstrated that overexpressed IDO-1 protein significantly ameliorates lung ischemia/ reperfusion injury (8). In this study, we showed that Tat-IDO-1 protein markedly reduced activation of microglia and astrocytes and reduced neuronal cell damages significantly in an ischemic injury animal model. Therefore, we suggest that IDO-1 protein may represent a potential therapeutic strategy against lung ischemia/reperfusion injury as well as brain ische- mic injury. However, further studies are needed to elucidate the exact protective mechanism on ischemic injury.

In summary, we showed that transduced Tat-IDO-1 protein inhibited oxidative stress-induced HT-22 cell death by reducing cellular cytotoxicity as well as regulation of cellular signaling pathways, such as apoptosis and MAPKs and Tat-IDO-1 protein transduced into the hippocampal CA1 region of the brain, and markedly ameliorates neuronal cell death. Therefore, Tat-IDO-1 protein can be a candidate as a useful therapeutic agent for ischemia.

\section{MATERIALS AND METHODS}

See supplementary information for this section.

\section{ACKNOWLEDGEMENTS}

This research was supported by the Basic Science Research Program through the National Research Foundation of Korea (NRF) funded by the Ministry of Education (2019R1A6A1A11036849).

\section{CONFLICTS OF INTEREST}

The authors have no conflicting interests.

\section{REFERENCES}

1. Tas SW, Vervoordeldonk MJ, Hajji $N$ et al (2007) Noncanonical NF-kappaB signaling in dendritic cells is required for indoleamine 2,3-dioxygenase (IDO) induction and immune regulation. Blood 1, 1540-1549

2. Uyttenhove C, Pilotte L, Theate I et al (2003) Evidence for a tumoral immune resistance mechanism based on tryptophan degradation by indoleamine 2,3-dioxygenase. Nat Med 9, 1269-1274

3. Wang $\mathrm{XF}$, Wang HS, Wang $\mathrm{H}$ et al (2014) The role of indoleamine 2,3-dioxygenase (IDO) in immune tolerance: focus on macrophage polarization of THP-1 cells. Cell Immunol 289, 42-48

4. Hirata F, Ohnishi T and Hayaish O (1977) Intracellular utilization of superoxide anion by indoleamine 2,3dioxygenase of rabbit enterocytes. J Biol Chem 252, 27741776

5. Chen Y and Guillemin GJ (2009) Kynurenine pathway metabolites in humans: Disease and healthy states. Int J Tryptophan Res 2, 1-19

6. Lugo-Huitron R, Blanco-Ayala $T$, Ugalde-Muniz $P$ et al (2011) On the antioxidant properties of kynurenic acid: free radical scavenging activity and inhibition of oxidative stress. Neurotoxicol Teratol 33, 538-547

7. Takikawa O (2005) Biochemical and medical aspects of the indoleamine 2,3-dioxygenase-initiated L-tryptophan metabolism. Biochem Biophys Res Commun 338, 12-19

8. Liu H, Liu L and Visner GA (2007) Nonviral gene delivery with indoleamine 2,3-dioxygenase targeting pulmonary endothelium protects against ischemia-reperfusion injury. Am J Transplant 10, 2291-2300 
9. Yun TJ, Lee JS, Machmach $K$ et al (2016) Indoleamine 2,3-dioxygenase-expressing aortic plasmacytoid dendritic cells protect against atherosclerosis by induction of regulatory T cells. Cell Metab 23, 852-866

10. Chen X, Guo C and Kong J (2012) Oxidative stress in neurodegenerative diseases. Neural Regen Res 7, 376-385

11. Kim GH, Kim JE, Rhie SJ and Yoon S (2015) The role of oxidative stress in neurodegenerative diseases. Exp Neurobiol 24, 325-340

12. Ashafaq M, Raza SS, Khan MM et al (2012) Catechin hydrate ameliorates redox imbalance and limits inflammatory response in focal cerebral ischemia. Neurochem Res 37, 1747-1760

13. Lei C, Deng J, Wang B et al (2011) Reactive oxygen species scavenger inhibits STAT3 activation after transient focal cerebral ischemia-reperfusion injury in rats. Anesth Analg 113, 153-159

14. Jeong HJ, Yoo DY, Kim DW et al (2014) Neuroprotective effect of PEP-1-peroxiredoxin2 on CA1 regions in the hippocampus against ischemic insult. Biochim Biophys Acta 1840, 2321-2330

15. Kim SM, Hwang IK, Yoo DY et al (2015) Tat-antioxidant 1 protects against stress-induced hippocampal $\mathrm{TH}-22$ cell death and ischemic insult in animal model. J Cell Mol Med 19, 1333-1345

16. Cheng J, Wang F, Yu DF, Wu PF and Chen JG (2011) The cytotoxic mechanism of malondialdehyde and protective effect of carnosine via protein cross-linking/ mitochondrial dysfunction/reactive oxygen species/MAPK pathway in neurons. Eur J Pharmacol 650, 184-194

17. Dhanasekaran DN and Reddy EP (2008) JNK signaling in apoptosis. Oncogene 27, 6245-6251

18. Kim SD, Moon CK, Eun SY, Ryu PD and Jo SA (2005) Identification of ASK1, MKK4, JNK, c-Jun, and caspase-3 as a signaling cascade involved in cadmium-induced neuronal cell apoptosis. Biochem Biophys Res Commun 328, 326-334

19. Ouyang $M$ and Shen $X$ (2006) Critical role of ASK1 in the 6-hydroxydopamine-induced apoptosis in human neuroblastoma SH-SY5Y cells. J Neurochem 97, 234-244

20. Wadia JS and Dowdy SF (2002) Protein transduction technology. Curr Opin Biotechnol 13, 52-56

21. Cerrato CP, Pirisinu M, Vlachos EN and Langel U (2015) Novel cell-penetrating peptide targeting mitochondria. FASEB J 29, 4589-4599

22. Jo HS, Yeo EJ, Shin MJ et al (2017) Tat-DJ-1 enhances cell survival by inhibition of oxidative stress, NF- $\mathrm{B} B$ and MAPK activation in HepG2 cells. Biotechnol Lett 39, 511521

23. Kim MJ, Park M, Kim DW et al (2015) Transduced PEP-1-PON1 proteins regulate microglial activation and dopaminergic neuronal death in a Parkinson's disease model. Biomaterials 64, 45-56

24. Gellert L, Fuzik J, Goblos A et al (2011) Neuroprotection with a new kynurenic acid analog in the four-vessel occlusion model of ischemia. Eur J Pharmacol 667, 182187

25. Lovelace MD, Varney B, Sundaram G et al (2017) Recent evidence for an expanded role of the kynurenine pathway of tryptophan metabolism in neurological diseases.
Neuropharmacol 112, 373-388

26. Duleu S, Mangas A, Sevin F, Veyret B, Bessede A and Geffard M (2010) Circulating antibodies to IDO/THO pathway metabolites in Alzheimer's disease. Int J Alzheimers Dis 2010, 6

27. Hu P, Hunt NH, Arfuso F et al (2017) Increased indoleamine 2,3-dioxygenase and quinolinic acid expression in microglia and muller cells of diabetic human and rodent retina. Invest Ophthalmol Vis Sci 58, 5043-5055

28. Taniguchi $T$, Sono $M$, Hirata $F$ et al (1979) Indoleamine 2,3-dioxygenase. Kinetic studies on the binding of superoxide anion and molecular oxygen to enzyme. J Biol Chem 254, 3288-3294

29. Sun $Y(1989)$ Indoleamine 2,3-dioxygenase-a new antioxidant enzyme. Mater Med Pol 21, 244-250

30. Freewan M, Rees MD, Plaza TS et al (2013). Human indoleamine 2,3-dioxygenase is a catalyst of physiological heme peroxidase reactions: implications for the inhibition of dioxygenase activity by hydrogen peroxide. J Biol Chem 288, 1548-1567

31. Ramsey JD and Flynn NH (2015) Cell-penetrating peptides transport therapeutics into cells. Pharmacol Ther 54, 78-86

32. Moon JI, Han MJ, Yu SH et al (2019) Enhanced delivery of protein fused to cell penetrating peptides to mammalian cells. BMB Rep 52, 324-329

33. Zhang X, Li Y, Cheng Y et al (2015) Tat PTD-endostatin: A novel anti-angiogenesis protein with ocular barrier permeability via eye-drops. Biochim Biophys Acta 1850, 1140-1149

34. Shin MJ, Kim DW, Lee YP et al (2014) Tat-glyoxalase protein inhibits against ischemic neuronal cell damage and ameliorates ischemic injury. Free Radic Biol Med 67, 195-210

35. Yeo HJ, Shin MJ, Yeo EJ et al (2019) Tat-CIAPIN1 inhibits hippocampal neuronal cell damage through the MAPK and apoptotic signaling pathways. Free Radic Biol Med $135,68-78$

36. Nakashima M, Niwa M, Iwai T and Uematsu T (1999) Involvement of free radicals in cerebral vascular reperfusion injury evaluated in a transient focal cerebral ischemia model of rat. Free Radic Biol Med 26, 722-729

37. Taguchi A, Hara A, Saito K et al (2008) Localization and spatiotemporal expression of IDO following transient forebrain ischemia in gerbils. Brain Res 1217, 78-85

38. Liang K, Ye Y, Wang Y, Zhang J and Li C (2014) Formononetin mediates neuroprotection against cerebral ischemia/reperfusion in rats via downregulation of the Bax/Bcl-2 ratio and upregulation PI3K/Akt signaling pathway. J Neurol Sci 344, 100-104

39. Zhang X, Shan P, Otterbein LE et al (2002) Carbon monoxide inhibition of apoptosis during ischemia-reperfusion lung injury is dependent on the p38 mitogen-activated protein kinase pathway and involves caspase 3. J Biol Chem 278, 1248-1258

40. Ferrer I, Lopez E, Blanco R et al (1998) Bcl-2, Bax, and $\mathrm{BCl}-\mathrm{x}$ expression in the CA1 area of the hippocampus following transient forebrain ischemia in the adult gerbil. Exp Brain Res 121, 167-173

41. Zhu N, Cai C, Zhou A, Zhao X, Xiang Y and Zeng C 
(2017) Schisandrin B prevents hind limb from ischemiareperfusion-induced oxidative stress and inflammation via MAPK/NF-kappaB pathways in Rats. Biomed Res Int 2017, 4237973

42. Kwon SH, Hong SI, Kim JA et al (2011) The neuroprotective effects of Lonicera japonica THUNB. against hydrogen peroxide-induced apoptosis via phosphorylation of MAPKs and PI3K/Akt in SH-SY5Y cells. Food Chem Toxicol 49, 1011-1019

43. Jia L, Chen Y, Tian YH and Zhang G (2018) MAPK pathway mediates the anti-oxidative effect of chicoric acid against cerebral ischemia-reperfusion injury in vivo. Exp Ther Med 15, 1640-1646

44. Guillemin GJ, Smythe G, Takikawa O and Brew BJ (2005) Expression of indoleamine 2,3-dioxygenase and production of quinolinic acid by human microglia, astrocytes, and neurons. Glia 49, 15-23

45. Grant RS, Naif H, Espinosa M and Kapoor V (2000) IDO induction in IFN-gamma activated astroglia: a role in improving cell viability during oxidative stress. Redox Rep 5, 101-104

46. Sugawara T, Lewen A, Noshita N, Gasche $Y$ and Chan PH (2002) Effects of global ischemia duration on neuronal, astroglial, oligodendroglial, and microglial reactions in the vulnerable hippocampal CA1 subregion in rats. J Neurotrauma 19, 85-98

47. Chu K, Yin B, Wang J et al (2012) Inhibition of P2X7 receptor ameliorates transient global cerebral ischemia/ reperfusion injury via modulating inflammatory responses in the rat hippocampus. J Neuroinflam 9, 69

48. Morizawa YM, Hirayama Y, Ohno N et al (2017) Reactive astrocytes function as phagocytes after brain ischemia via ABCA1-mediated pathway. Nat Commun 8, 28

49. Annunziato L, Boscia F and Pignataro G (2013) Ionic transporter activity in astrocytes, microglia, and oligodendrocytes during brain ischemia. J Cereb Blood Flow Metab 33, 969-982 